\title{
Derivation of characteristics of the relation between geomagnetic and geoelectric variation fields from the surface impedance for a two-layer earth
}

\author{
Risto Pirjola \\ Finnish Meteorological Institute, P. O. Box 503, FI-00101 Helsinki, Finland \\ (Received March 25, 2009; Revised May 13, 2009; Accepted September 26, 2009; Online published March 4, 2010)
}

\begin{abstract}
The surface impedance is defined to give the ratio between horizontal geoelectric and geomagnetic variation fields at the earth's surface in the frequency domain. Studying the properties of the surface impedance enables conclusions about the corresponding relation between the surface electric and magnetic variation fields in the time domain. In particular, it is possible to perform an investigation about assumptions that lead to a proportionality between the geoelectric field and the time derivative of the geomagnetic field and about situations that make the electric field and the variations of the magnetic field proportional. The results are directly applicable to the research of geomagnetically induced currents (GIC) driven by the geoelectric field in technological networks at the earth's surface. Thus, the main objective of this paper is not in traditional magnetotellurics but in studies of ground effects of space weather. We use a two-layer earth model, which is simple enough to have a precise analytic formula for the surface impedance. It is shown in this paper that a poorly-conducting upper layer above a highly-conducting bottom is favourable to the electric field (and GIC) being proportional to the magnetic time derivative at the earth's surface whereas a thin highly-conducting upper layer above a less-conducting bottom results in a surface electric field (and GIC) proportional to magnetic variations.
\end{abstract}

Key words: Surface impedance, geoelectric field, geomagnetic field, geomagnetically induced current, GIC, space weather, plane wave, layered earth.

\section{Introduction}

At the earth's surface space weather, which is controlled by solar activity, manifests itself as geomagnetically induced currents (GIC) in technological networks, such as electric power transmission grids, oil and gas pipelines, telecommunication cables and railway equipment (e.g. Boteler et al., 1998). In general, GIC are a possible source of problems in the particular system. In power networks, this is due to saturation of transformers (e.g. Kappenman, 2007). GIC are driven by the horizontal geoelectric field induced by a time variation of the geomagnetic field at the earth's surface. So physically, the generation of GIC is governed by Faraday's and Ohm's laws. Since the geoelectric field is the key quantity for GIC, research efforts need to be focussed on understanding the behaviour of the geoelectric field and on its determination techniques. The production of GIC by the geoelectric field is (approximately) a dc process, which means that GIC follow the time variations of the electric field. Thus, the issues discussed in this paper about the behaviour of the electric field are directly applicable to GIC as well. Consequently, the principal objective of this paper is related to the research of GIC, i.e. ground effects of space weather, and not to traditional magnetotelluric and geoelectromagnetic induction studies

Copyright (C) The Society of Geomagnetism and Earth, Planetary and Space Sciences (SGEPSS); The Seismological Society of Japan; The Volcanological Society of Japan; The Geodetic Society of Japan; The Japanese Society for Planetary Sciences; TERRAPUB.

doi:10.5047/eps.2009.09.002 performed intensively for several decades. In other words, this paper attempts to fill the gap between the space weather and magnetotelluric communities.

Since the geoelectric field is induced by a geomagnetic variation, it is natural that the electric field is related to the time derivative of the magnetic field. The first guess is that these two quantities would be proportional. However, the geoelectromagnetic field is produced by primary currents in the ionosphere and magnetosphere and by secondary currents and charges induced in the conducting earth as described by Maxwell's equations and boundary conditions. The result from this complicated process is that the dependence of the electric field on the magnetic time derivative is generally not a simple proportionality. Even in the most simple case modelled by vertically propagating plane waves and a uniform half-space earth, the electric field at a given time is obtained from an integral including all past values of the time derivative of the magnetic field (Cagniard, 1953; Pirjola, 1982).

Trichtchenko and Boteler $(2006,2007)$ present an example from Canada in which GIC resembles the geomagnetic variation and another example also from Canada in which a correspondence between GIC and the magnetic time derivative exists. Watari et al. (2009) demonstrate that in recordings in the Japanese power network GIC show a high correlation with the geomagnetic variation field rather than with the magnetic time derivative. In this paper, we perform a theoretical study of the relation between the surface elec- 
tric and magnetic variation fields and look for conditions concerning the conductivity structure of the earth that determine whether the electric field has a close connection to the magnetic variation or to the magnetic time derivative.

The study is made by considering the surface impedance, which is defined as the transfer function between the geoelectric and geomagnetic variation fields at the earth's surface (e.g. Kaufman and Keller, 1981). In order to keep the equations tractable, we assume that the earth, which is described by a half-space, only has two layers, i.e. an upper layer above an infinite bottom. This is naturally an idealisation but allows us to derive features important for the relation between the electric and magnetic fields and particular useful for basic GIC research. Another assumption is that we have the "plane wave case", which is satisfied for a primary field that is a vertically downward propagating plane wave. In practice, this assumption may lead to incorrect results only in high-latitude auroral regions. The concept of the surface impedance, or equivalently of the magnetotelluric response function, is very well-known and widely used in magnetotelluric investigations of the structure of the earth (e.g. Cagniard, 1953; Schmucker, 1970; Weidelt, 1972; Berdichevsky and Dmitriev, 1976; Szarka and Fischer, 1989; Weaver, 1994; Szarka, 1997; and many others). Pirjola et al. (2009) provide a review of the use of the surface impedance in connection with research of ground effects of space weather.

\section{Surface Impedance}

Choosing the standard Cartesian $x y z$ coordinate system in which the earth's surface is the $x y$ plane and the $x, y$ and $z$ axes point northwards, eastwards and downwards, the (scalar) surface impedance $Z$ is defined by

$$
Z=-\mu_{0} \frac{E_{y}}{B_{x}}
$$

where $E_{y}$ and $B_{x}$ are perpendicular horizontal electric and magnetic components at the earth's surface. To be precise, $B_{x}$ only refers to the magnetic variation field, i.e. not to the total geomagnetic field, which is a remark valid throughout this paper. The minus sign and the vacuum permeability $\mu_{0}=4 \pi \cdot 10^{-7} \mathrm{~V} \mathrm{~s} \mathrm{~A}^{-1} \mathrm{~m}^{-1}$, which gives the unit of 'ohm' $[\Omega]$ to the surface impedance, are included in Eq. (1) for convenience. This formula can be regarded as a general definition independently of the domains (time, frequency, space, wavenumber) in which $E_{y}$ and $B_{x}$ are considered. Most commonly, however, the electric and magnetic components used in Eq. (1) are Fourier transforms in the frequency and wavenumber domains. Then $E_{y}$ and $B_{x}$ are generally complex-valued making $Z$ complex, too. A recursive formula for the surface impedance in the frequency and wavenumber domains can be derived for a layered earth in a straightforward manner by using the electromagnetic continuity conditions at the layer boundaries (e.g. Wait, 1981, pp. 43-55).

Assuming that the primary electromagnetic field is a plane wave propagating vertically and incident on a layered earth, both the secondary reflected field in the air and the fields in the earth layers also propagate vertically and no dependence on $x$ or $y$ exists. We now restrict the dis- cussion to this "plane wave case" and assume that $E_{y}$ and $B_{x}$ are Fourier transformed from the time $(t)$ to the angular frequency $(\omega)$ domain. Consequently, besides the earth's structure, the surface impedance only depends on the angular frequency $\omega$. Thus, rewriting Eq. (1),

$$
E_{y}(\omega)=-\frac{1}{\mu_{0}} Z_{\mathrm{p}}(\omega) B_{x}(\omega)
$$

where the subscript ' $p$ ' refers to 'plane wave'. Denoting the time derivative of $B_{x}(t)$ by $g(t)\left(=d B_{x}(t) / d t\right)$ and using the relation $g(\omega)=i \omega B_{x}(\omega)$, Eq. (2) gives

$$
E_{y}(\omega)=-\frac{Z_{\mathrm{p}}(\omega)}{i \omega \mu_{0}} g(\omega)
$$

Inverse Fourier transforming Eqs. (2) and (3) from $\omega$ to $t$, we obtain the relations between the electric and magnetic fields in the time domain, which are convolution integrals (see Pirjola et al., 2009). Equation (2) directly indicates that if $Z_{\mathrm{p}}$ is independent of $\omega, E_{y}(t)$ is proportional to $B_{x}(t)$. Similarly from Eq. (3), if $Z_{\mathrm{p}}$ is a linear function of $\omega E_{y}(t)$ is proportional to $g(t)$.

Let us now assume that the earth is composed of two layers, i.e. an upper layer with a thickness $h$ above an infinite bottom. Denote the permeability, permittivity and conductivity of the upper layer by $\mu_{1}, \varepsilon_{1}$ and $\sigma_{1}$, respectively. The corresponding parameters of the bottom are $\mu_{2}, \varepsilon_{2}$ and $\sigma_{2}$. As shown e.g. by Pirjola (1982), the surface impedance is then

$$
Z_{\mathrm{p}}=\frac{\mu_{1} \omega \alpha_{2}}{k_{1} \alpha_{1}}
$$

where $k_{1}$ is the propagation constant of the upper layer, given below, and $\alpha_{1}$ and $\alpha_{2}$ are defined as follows:

$$
\alpha_{1}=1+\frac{\mu_{1} k_{2}-\mu_{2} k_{1}}{\mu_{1} k_{2}+\mu_{2} k_{1}} e^{-2 i k_{1} h}
$$

and

$$
\alpha_{2}=1-\frac{\mu_{1} k_{2}-\mu_{2} k_{1}}{\mu_{1} k_{2}+\mu_{2} k_{1}} e^{-2 i k_{1} h}
$$

The propagation constants $k_{1}$ and $k_{2}$ are given by

$$
k_{n}=\sqrt{\omega^{2} \mu_{n} \varepsilon_{n}-i \omega \mu_{n} \sigma_{n}} \quad(n=1,2)
$$

In practical geoelectromagnetic situations $\sigma_{n}$ is much larger than $\omega \varepsilon_{n}$, and so

$$
k_{n}=\sqrt{-i \omega \mu_{n} \sigma_{n}}
$$

The application of Eq. (8) involves the neglect of displacement currents in the earth, which implies diffusion of the electromagnetic field, contrary to wave propagation that occurs in the upper half-space, the air. For simplicity, let us assume that $\mu_{1}=\mu_{2}=\mu_{0}$. Then

$$
\alpha_{1}=1+\beta
$$

and

$$
\alpha_{2}=1-\beta
$$

where

$$
\beta=\frac{k_{2}-k_{1}}{k_{2}+k_{1}} e^{-2 i k_{1} h}
$$


A few additional words are needed associated with the equations presented above. As indicated above, a formula for the surface impedance can be derived based on electromagnetic boundary conditions. Thus Eq. (4), which does not involve any approximations, is directly and easily obtained by considering upward and downward propagating plane waves in the media and by using boundary conditions at $z=0$ and $z=h$. The two-layer case included in Eq. (4) has been discussed in magnetotelluric literature many times, even in the basic magnetotelluric paper by Cagniard (1953). Generally, the reasonable approximations of formula (8) and of equal permeabilities are used in the equation for the surface impedance that corresponds to formula (4) (see e.g. Kaufman and Keller, 1981, equation (2.43); Szarka, 1997, equation (A.13)). Equivalently to the surface impedance, the magnetotelluric response function $c=c(\omega)=Z_{\mathrm{p}}(\omega) /\left(i \omega \mu_{0}\right)$ can be used as well. Consequently, for example, equation (3.93) of Weaver (1994) is comparable to Eq. (4). It is worth noting that the magnetotelluric response function is sometimes known as the complex (skin) depth (Szarka and Fischer, 1989). This is especially the case in connection with GIC studies (Boteler and Pirjola, 1998; Pirjola and Viljanen, 1998). The surface impedance, the magnetotelluric response function and the complex skin depth can be regarded as mathematical concepts that help modelling studies and interpretation of data but, as presented by Szarka and Fischer (1989), analyses of the real and imaginary parts of these quantities yield information about the locations of earth currents that are in phase or out of phase with respect to the surface magnetic variation.

\section{Case 1: Poorly-Conducting Surface Layer Above Highly-Conducting Bottom}

We assume that $\sigma_{2} \gg \sigma_{1}$ and $h \ll\left|1 / k_{1}\right|$. The extreme situation that satisfies these assumptions is a perfect conductor underlying a non-conducting surface layer. Defining the skin depth $\delta_{n}(n=1,2)$ as

$$
\delta_{n}=\sqrt{\frac{2}{\omega \mu_{n} \sigma_{n}}}
$$

and taking Eq. (8) into account, the latter assumption means that the skin depth of the upper layer is much larger than its thickness. The absolute value of the exponent in the function $e^{-2 i k_{1} h}$ is thus very small, and so the function can be approximated by $1-2 i k_{1} h$ Utilising the assumption $\sigma_{2} \gg \sigma_{1}$ and Eq. (8), we may consider $\left|k_{2}\right|$ much larger than $\left|k_{1}\right|$. Consequently from Eq. (11),

$$
\beta \approx e^{-2 i k_{1} h} \approx 1-2 i k_{1} h
$$

leading, based on Eqs. (9) and (10), to

$$
\alpha_{1} \approx 2-2 i k_{1} h \approx 2
$$

and

$$
\alpha_{2} \approx 2 i k_{1} h
$$

Substituting Eqs. (14) and (15) into formula (4) (with $\mu_{1}=$ $\left.\mu_{0}\right)$, we obtain

$$
Z_{\mathrm{p}} \approx i \omega \mu_{0} h
$$

Thus $Z_{\mathrm{p}}$ is a linear function of $\omega$, and so $E_{y}(t)$ is proportional to $g(t)$ (see Section 2). Equations (3) and (16) give (after an inverse Fourier transform to the time domain)

$$
E_{y}(t)=-h g(t)
$$

Equations (2) and (16) demonstrate that, for the present earth model, there is a ninety-degree phase shift between $-E_{y}$ and $B_{x}$ in the frequency domain, which is a wellknown result in magnetotellurics. Furthermore, it should be noted that Eq. (16) corresponds to the approximation of equation (3.99) of Weaver (1994) by the first term on its right-hand side.

In conclusion, if the earth is composed of a poorlyconducting upper layer above a highly-conducting bottom the horizontal electric field is proportional to the time derivative of the perpendicular horizontal magnetic field at the earth's surface. Pirjola and Viljanen (1989) discuss the extreme earth model by assuming that there is a nonconducting layer above a perfect conductor, and they show explicitly the proportionality between the electric field and the magnetic time derivative. In fact, their study does not refer to the plane wave case but they consider an ionospheric line current source, which makes the proportionality factor, as well as the electric field and the magnetic time derivative, vary from site to site at the earth's surface.

Equation (17) shows that an increase of the thickness $h$ of the upper layer has an enhancing effect on the electric field. By noting that an increase of $h$ means that the poorlyconducting part of the earth gets more "weight" the observation is in agreement with the fact that a more resistive earth is favourable to large geoelectric fields.

It is worth pointing out that Berdichevsky and Dmitriev (1976) already derived Eq. (16) in connection with analyses of magnetotelluric sounding curves. They considered a three-layer earth model in which the infinite bottom is perfectly-conducting, the middle layer is non-conducting and the surface layer has finite conductivity. When the period is large enough, i.e. $\omega$ small enough, the surface layer does not have much influence and we have a poor conductor above a highly-conducting bottom. This leads to Eq. (16) as seen in the asymptotic formula on page 175 of Berdichevsky and Dmitriev (1976).

\section{Case 2: Thin Highly-Conducting Surface Layer Above Poorly-Conducting Bottom}

Let us assume now that $\sigma_{1} \gg \sigma_{2}$ and $h \ll\left|1 / k_{1}\right|$. Note that, differently from Case 1 in Section 3, these assumptions are a little contradictory because the first assumption requires that the upper layer is highly conducting whereas the second assumption does not allow the conductivity of the upper layer be so high that the skin depth is reduced to be comparable with the thickness $h$. (This is the reason for inserting the word "thin" into the title of this section.) The assumption $\sigma_{1} \gg \sigma_{2}$ leads to $\left|k_{1}\right| \gg\left|k_{2}\right|$ (Eq. (8)), and so with the assumption $h \ll\left|1 / k_{1}\right|$ Eq. (11) yields

$$
\beta \approx-e^{-2 i k_{1} h} \approx-1+2 i k_{1} h
$$

Thus Eqs. (9) and (10) give

$$
\alpha_{1} \approx 2 i k_{1} h
$$




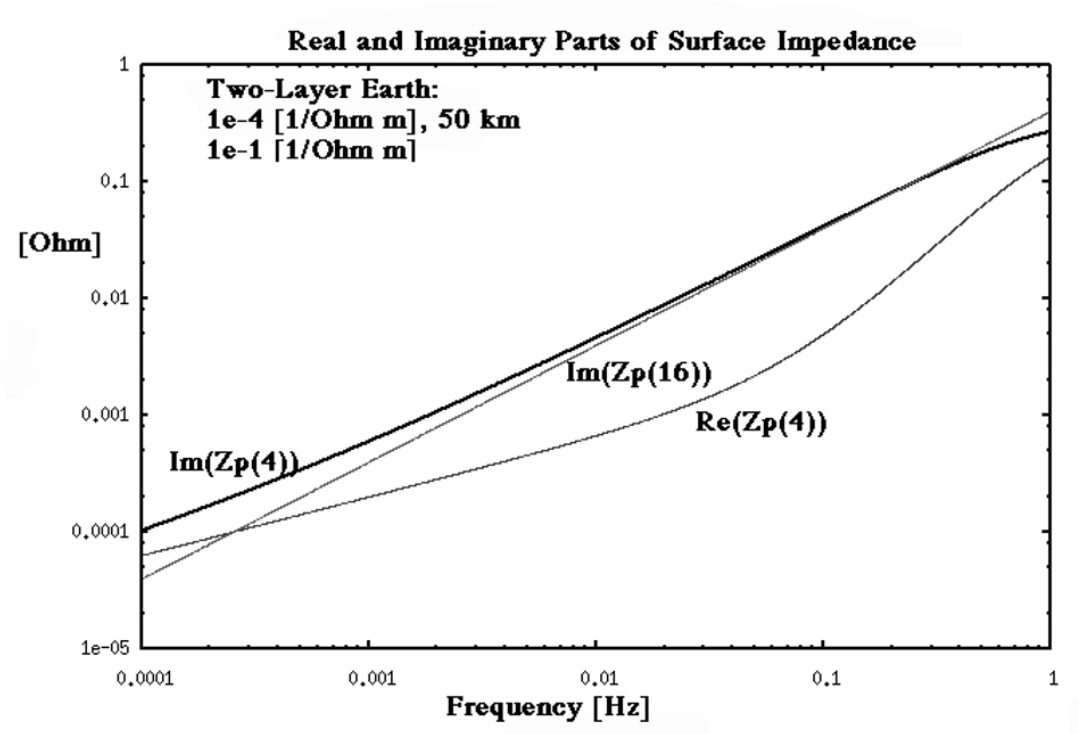

Fig. 1. Real and imaginary parts of the exact surface impedance given by Eq. (4) for a two-layer earth, and the imaginary part of the approximate surface impedance given by Eq. (16). The thickness of the upper layer is $50 \mathrm{~km}$. The conductivities of the upper layer and of the bottom are $10^{-4} \Omega^{-1} \mathrm{~m}^{-1}$ and $10^{-1} \Omega^{-1} \mathrm{~m}^{-1}$, respectively. The quantities are plotted as functions of the frequency $(=\omega / 2 \pi)$.

and

$$
\alpha_{2} \approx 2-2 i k_{1} h \approx 2
$$

Substituting Eqs. (19) and (20) into formula (4) (with $\mu_{1}=$ $\mu_{0}$ ) and using Eq. (8) (with $n=1$ ), we obtain

$$
Z_{\mathrm{p}} \approx \frac{1}{\sigma_{1} h}
$$

Thus $Z_{\mathrm{p}}$ is independent of the angular frequency $\omega$, and so $E_{y}(t)$ is proportional to $B_{x}(t)$ (see Section 2). Equations (2) and (21) give (after an inverse Fourier transform to the time domain)

$$
E_{y}(t)=-\frac{1}{\mu_{0} \sigma_{1} h} B_{x}(t)
$$

Equations (2) and (21) demonstrate that, for the present earth model, $-E_{y}$ and $B_{x}$ do not have any phase shift in the frequency domain, which is a well-known result in magnetotellurics. Furthermore, it should be noted that Eq. (21) corresponds to the approximation of equation (3.95) of Weaver (1994) by the second term of its middle form.

In conclusion, if the earth is composed of a highlyconducting upper layer above a poorly-conducting bottom the time behaviour of the horizontal electric field follows that of the perpendicular horizontal magnetic variation at the earth's surface.

Equation (22) shows that an increase of the thickness $h$ of the upper layer has a decreasing effect on the electric field. By noting that an increase of $h$ means that the highlyconducting part of the earth gets more "weight" the observation is in agreement with the fact that a more conducting earth is not so favourable to large geoelectric fields.

Let us finally note that, similarly to Case 1 discussed in Section 3, Eq. (21) was also already derived by Berdichevsky and Dmitriev (1976) by considering their three-layer earth model. When the period is small enough, i.e. $\omega$ large enough, the surface layer does have an influence and we have a conducting layer above a poor conductor. This leads to Eq. (21) as seen in the asymptotic formula on page 175 of Berdichevsky and Dmitriev (1976).

\section{Numerical Examples}

In this section we examine the validity of the approximations expressed by formulas (16) and (21) for the surface impedance $Z_{\mathrm{p}}$ given exactly by Eq. (4). It should be noted that the real part of $Z_{\mathrm{p}}(16)$ and the imaginary part of $Z_{\mathrm{p}}(21)$ are zero. Moreover, $Z_{\mathrm{p}}(21)$ does not depend on the frequency at all. Let us consider the frequency range from $f(=\omega / 2 \pi)=10^{-4} \mathrm{~Hz}$ to $f=1 \mathrm{~Hz}$ important in geoelectromagnetics.

Figure 1 presents the real and imaginary parts of the exact surface impedance $Z_{\mathrm{p}}(4)$ together with the imaginary part of the approximate surface impedance $Z_{\mathrm{p}}(16)$ when $h=50 \mathrm{~km}, \sigma_{1}=10^{-4} \Omega^{-1} \mathrm{~m}^{-1}$ and $\sigma_{2}=10^{-1} \Omega^{-1}$ $\mathrm{m}^{-1}$. These parameter choices refer to Case 1 (Section 3 ) but at the larger frequencies considered the assumption $h \ll$ $\left|1 / k_{1}\right|$ is not valid. Figure 2 shows the real and imaginary parts of the exact surface impedance $Z_{\mathrm{p}}(4)$ together with (the real part of) the approximate surface impedance $Z_{\mathrm{p}}(21)$ when $h=10 \mathrm{~km}, \sigma_{1}=10^{-2} \Omega^{-1} \mathrm{~m}^{-1}$ and $\sigma_{2}=10^{-5} \Omega^{-1}$ $\mathrm{m}^{-1}$. These parameter choices refer to Case 2 (Section 4 ) but again at the larger frequencies considered the assumption $h \ll\left|1 / k_{1}\right|$ is not valid. Figure 3 contains the same information as Figs. 1 and 2 by presenting the absolute values (3(a)) and phase angles (3(b)) of the surface impedances. In Fig. 3(b), we naturally only have the curves for the exact surface impedance $Z_{\mathrm{p}}(4)$ because the phases of $Z_{\mathrm{p}}(16)$ and $Z_{\mathrm{p}}(21)$ are 90 degrees and zero, respectively. It can be noted that the curve labeled " $\left|Z_{\mathrm{p}}(4)\right|$ (Fig. 2)" is the same as briefly analysed by Pirjola et al. (2009). They emphasise the small variation of the absolute value of the surface impedance as an indication of the electric field to be roughly proportional to the magnetic field in this case. They, how- 


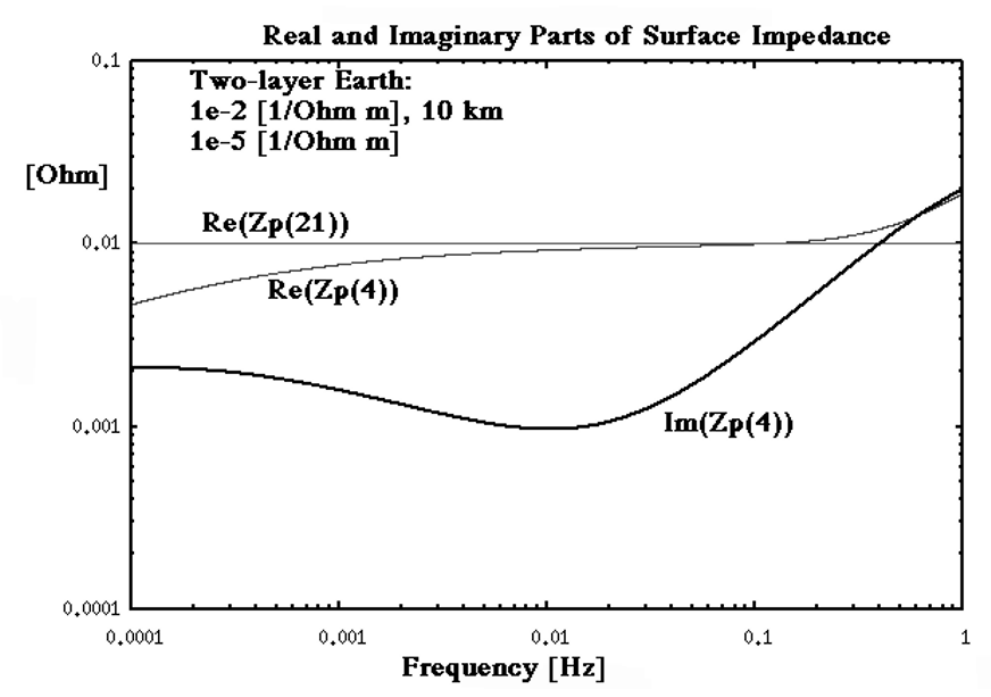

Fig. 2. Real and imaginary parts of the exact surface impedance given by Eq. (4) for a two-layer earth, and (the real part of) the approximate surface impedance given by Eq. (21). The thickness of the upper layer is $10 \mathrm{~km}$. The conductivities of the upper layer and of the bottom are $10^{-2} \Omega^{-1} \mathrm{~m}^{-1}$ and $10^{-5} \Omega^{-1} \mathrm{~m}^{-1}$, respectively. The quantities are plotted as functions of the frequency $(=\omega / 2 \pi)$.

(a)

Absolute Value of Surface Impedance

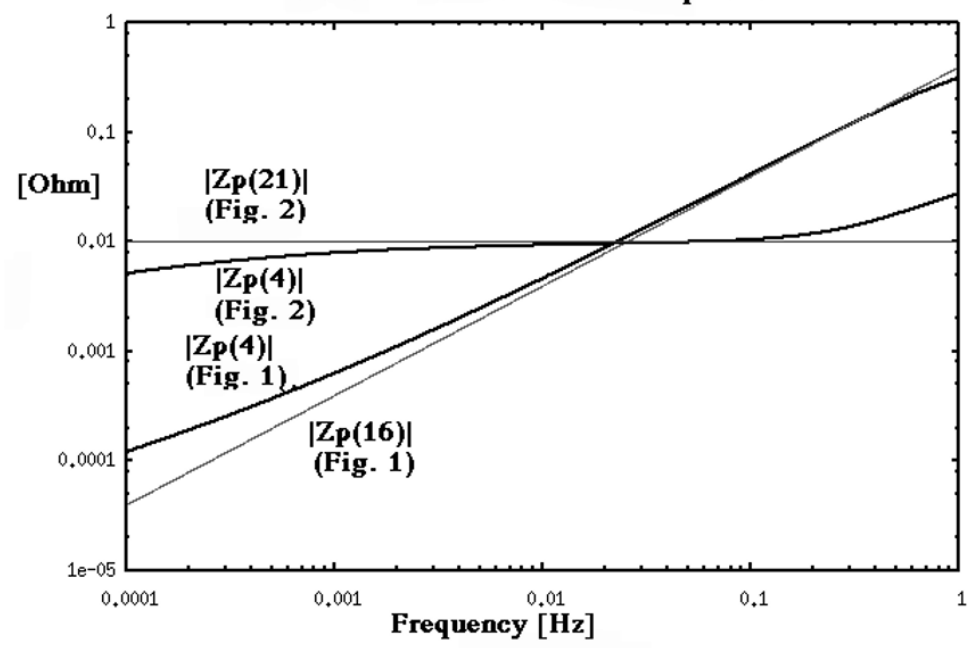

(b)

Phase Angle of Surface Impedance

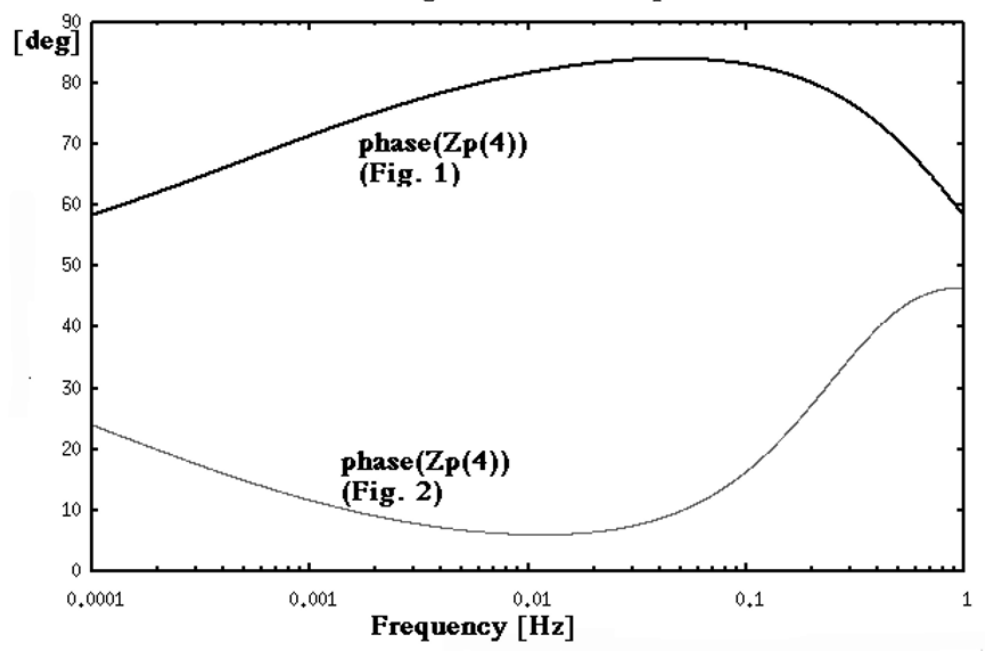

Fig. 3. (a): Absolute values of the surface impedances presented in Figs. 1 and 2. (b): Phase angles of the exact surface impedances (Eq. (4)) presented in Figs. 1 and 2. The phase angles of the approximate surface impedances obtained from Eqs. (16) and (21) are 90 degrees and zero, respectively. The quantities are plotted as functions of the frequency $(=\omega / 2 \pi)$. 


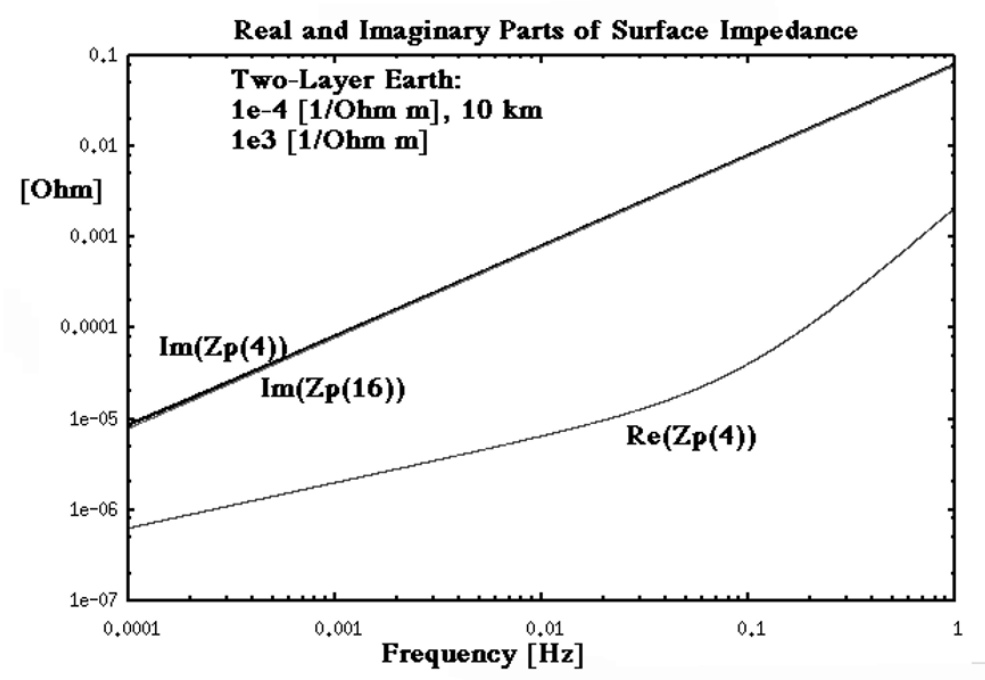

Fig. 4. Real and imaginary parts of the exact surface impedance given by Eq. (4) for a two-layer earth, and the imaginary part of the approximate surface impedance given by Eq. (16). The thickness of the upper layer is $10 \mathrm{~km}$. The conductivities of the upper layer and of the bottom are $10^{-4} \Omega^{-1} \mathrm{~m}^{-1}$ and $1000 \Omega^{-1} \mathrm{~m}^{-1}$, respectively. The quantities are plotted as functions of the frequency $(=\omega / 2 \pi)$.

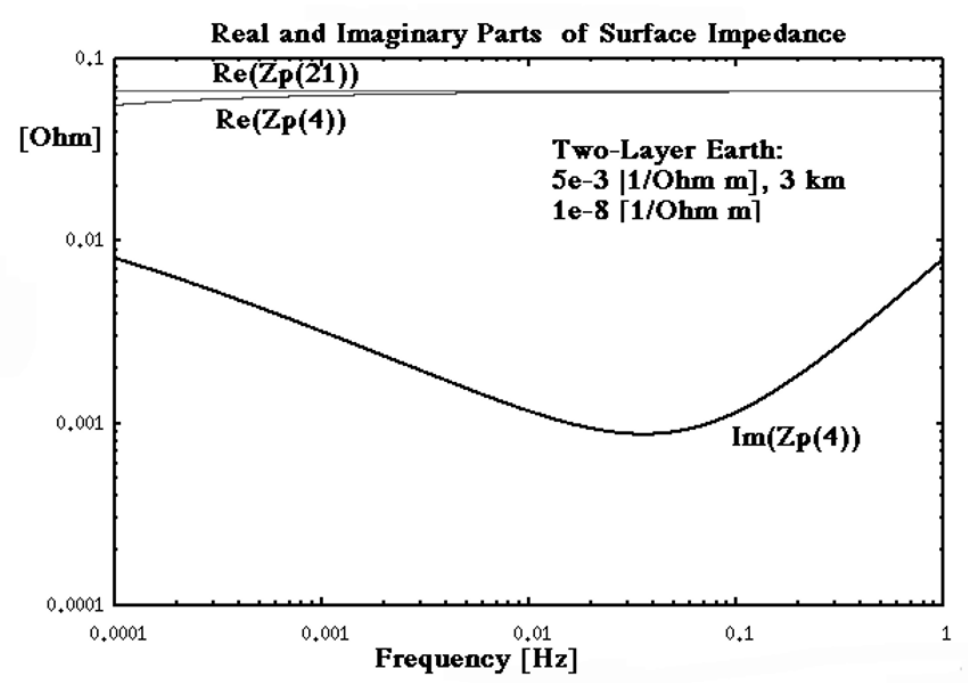

Fig. 5. Real and imaginary parts of the exact surface impedance given by Eq. (4) for a two-layer earth, and (the real part of) the approximate surface impedance given by Eq. (21). The thickness of the upper layer is $3 \mathrm{~km}$. The conductivities of the upper layer and of the bottom are $5 \cdot 10^{-3} \Omega^{-1} \mathrm{~m}^{-1}$ and $10^{-8} \Omega^{-1} \mathrm{~m}^{-1}$, respectively. The quantities are plotted as functions of the frequency $(=\omega / 2 \pi)$.

ever, do not consider the phase angle at all.

The agreement between $Z_{\mathrm{p}}(4)$ and $Z_{\mathrm{p}}(16)$ presented in Fig. 1 is the best in the middle part of the frequency range considered. Similarly the agreement between $Z_{p}(4)$ and $Z_{\mathrm{p}}(21)$ shown in Fig. 2 is also the best in the middle range. These conclusions are confirmed by Fig. 3. The obvious reason for the discrepancies at the large frequencies is in the above-mentioned invalidity of the assumption $h \ll\left|1 / k_{1}\right|$. But understanding the low-frequency behaviour constitutes a bigger problem. Looking at the derivations of Eqs. (16) and (21) more carefully, we notice that they utilise the approximations included in Eqs. (15) and (19). Due to the approximation $h \ll\left|1 / k_{1}\right|$, which is satisfied very well when $\omega$ is small, $\alpha_{2}$ given by Eq. (15) and $\alpha_{1}$ given by Eq. (19) are very small at the lowest frequencies. Thus the calculations presented in Sections 3 and 4 are not correct if terms larger than the particular value of $\alpha_{2}$ or $\alpha_{1}$ are dropped away during the approximations.

Consequently, let us consider the derivation of Eq. (15) more precisely. We obtain from Eqs. (10) and (11)

$$
\begin{aligned}
\alpha_{2} & =1-\beta=1-\frac{k_{2}-k_{1}}{k_{2}+k_{1}} e^{-2 i k_{1} h} \\
& \approx 1-\frac{k_{2}-k_{1}}{k_{2}+k_{1}}\left(1-2 i k_{1} h\right) \\
& =\frac{2 k_{1}}{k_{2}+k_{1}}+\frac{k_{2}-k_{1}}{k_{2}+k_{1}} 2 i k_{1} h
\end{aligned}
$$

To get formula (15), we need to neglect the first term $2 k_{1} /\left(k_{2}+k_{1}\right)$ and set $\left(k_{2}-k_{1}\right) /\left(k_{2}+k_{1}\right)$ equal to one in the second term. However, neglecting the term $2 k_{1} /\left(k_{2}+k_{1}\right)$ is not acceptable if its absolute value is of the same order of 

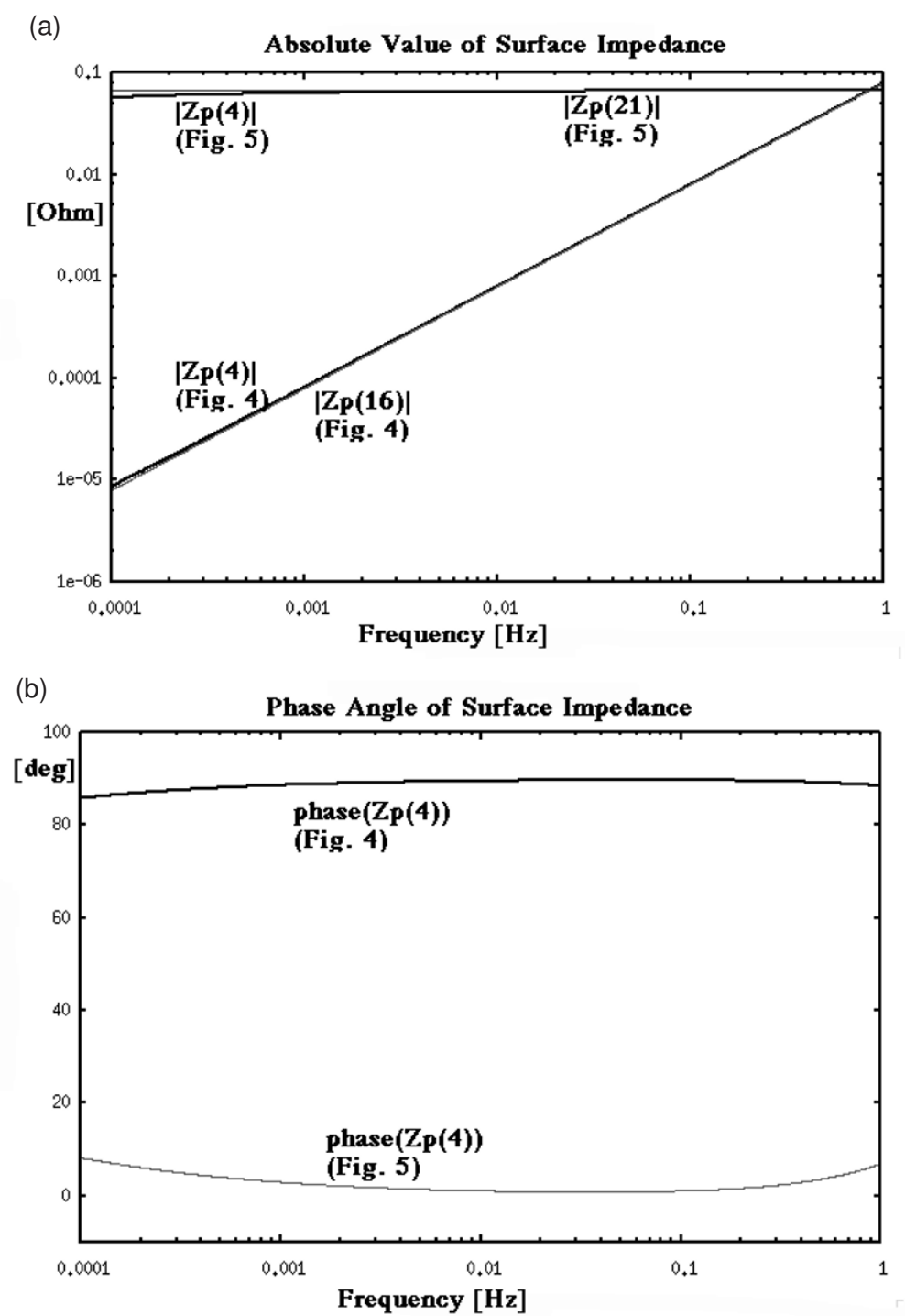

Fig. 6. (a): Absolute values of the surface impedances presented in Figs. 4 and 5. (b): Phase angles of the exact surface impedances (Eq. (4)) presented in Figs. 4 and 5. The phase angles of the approximate surface impedances obtained from Eqs. (16) and (21) are 90 degrees and zero, respectively. The quantities are plotted as functions of the frequency $(=\omega / 2 \pi)$.

magnitude or larger than the absolute value of the remaining term $2 i k_{1} h$, which is very small, as indicated above. Thus the use of Eq. (15) requires that $\left|k_{1}\right|$ is really very much smaller than $\left|k_{2}\right|$ making the neglected term $2 k_{1} /\left(k_{2}+k_{1}\right)$ extremely small, i.e. the conductivity of the bottom has to be enormous relative to that of the upper layer. This requirement is not satisfied by the chosen parameter values, which thus explains the disagreement between $Z_{\mathrm{p}}(4)$ and $Z_{\mathrm{p}}(16)$ at the small frequencies in Figs. 1 and 3. Exactly in the same way, the use of Eq. (19) is allowed provided that the conductivity of the upper layer is extremely large in comparison with that of the bottom, and due to the invalidity of this requirement, $Z_{\mathrm{p}}(4)$ and $Z_{\mathrm{p}}(21)$ do not agree in Figs. 2 and 3 at the low frequencies. It should be stressed that the approximations included in Eqs. (14) and (20) do not suffer from the same problem because the remaining term $(\approx 2)$ is not too small.

We now test the justification of this reasoning by considering unrealistically large conductivity contrasts between the upper layer and the bottom. Figure 4 presents the real and imaginary parts of the exact surface impedance $Z_{p}(4)$ together with the imaginary part of the approximate surface impedance $Z_{\mathrm{p}}(16)$ when $h=10 \mathrm{~km}, \sigma_{1}=10^{-4} \Omega^{-1} \mathrm{~m}^{-1}$ and $\sigma_{2}=1000 \Omega^{-1} \mathrm{~m}^{-1}$. These parameter choices refer to Case 1 (Section 3), and the assumption $h \ll\left|1 / k_{1}\right|$ is satisfied though not very well at the largest frequencies. Figure 5 shows the real and imaginary parts of the exact surface impedance $Z_{\mathrm{p}}(4)$ together with (the real part of) the approximate surface impedance $Z_{\mathrm{p}}(21)$ when $h=3 \mathrm{~km}$, $\sigma_{1}=5 \cdot 10^{-3} \Omega^{-1} \mathrm{~m}^{-1}$ and $\sigma_{2}=10^{-8} \Omega^{-1} \mathrm{~m}^{-1}$. These parameter choices refer to Case 2 (Section 4 ), and the assumption $h \ll\left|1 / k_{1}\right|$ is satisfied though again not well at the largest frequencies. Figure 6, which corresponds to Fig. 3, contains the same information as Figs. 4 and 5 by presenting the absolute values (6(a)) and phase angles (6(b)) of the surface impedances. Figures 4, 5 and 6 demonstrate that the selected conductivity and thickness values make the approximate formulas (16) and (21) of the surface impedance quite accurate, and so the above arguments about the reasons for the failure of the approximations in Figs. 1, 2 and 


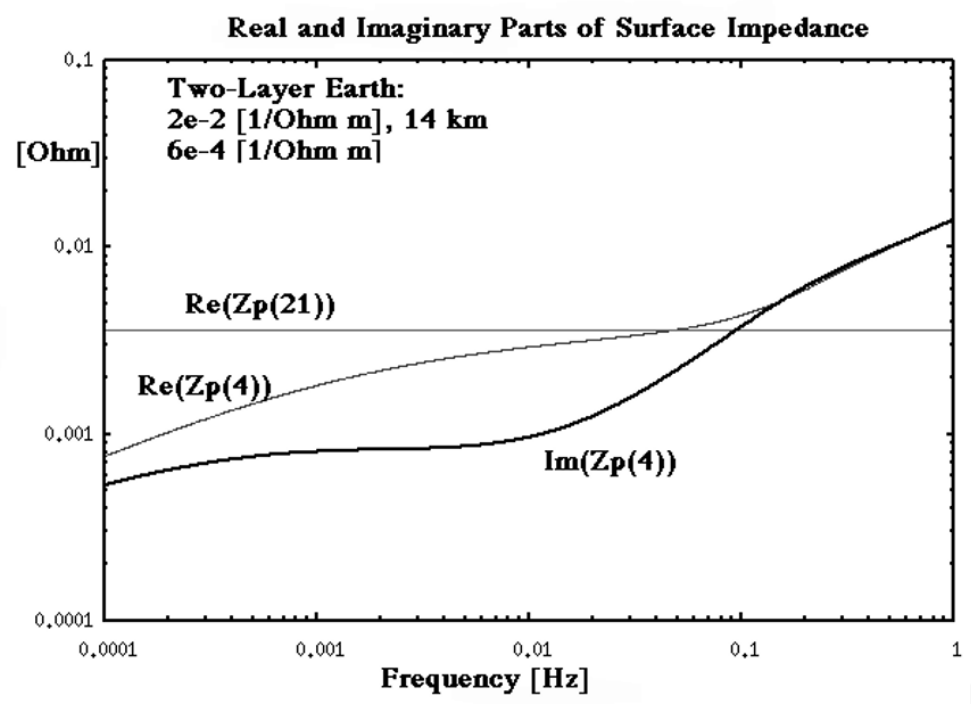

Fig. 7. Real and imaginary parts of the exact surface impedance given by Eq. (4) for a two-layer earth, and (the real part of) the approximate surface impedance given by Eq. (21). The thickness of the upper layer is $14 \mathrm{~km}$. The conductivities of the upper layer and of the bottom are $2 \cdot 10^{-2} \Omega^{-1} \mathrm{~m}^{-1}$ and $6 \cdot 10^{-4} \Omega^{-1} \mathrm{~m}^{-1}$, respectively. These numerical values are adopted based on Watari et al. (2009). The quantities are plotted as functions of the frequency $(=\omega / 2 \pi)$.

3 are shown to be correct.

As mentioned in Section 1, studies by Watari et al. (2009) show a correlation of time variations of GIC with those of the geomagnetic field in Japan. The three-layer earth models mentioned by Watari et al. (2009) for the area of their research have the highest conductivity at the top. Thus based on Case 2 discussed in Section 4, the observed correlation between GIC and the magnetic field is understandable. Let us investigate the issue in greater detail. For our two-layer model we adopt the values $h=14 \mathrm{~km}$, $\sigma_{1}=2 \cdot 10^{-2} \Omega^{-1} \mathrm{~m}^{-1}$ and $\sigma_{2}=6 \cdot 10^{-4} \Omega^{-1} \mathrm{~m}^{-1}$, which roughly approximate the numbers given by Watari et al. (2009). Figure 7 depicts the real and imaginary parts of the exact surface impedance $Z_{\mathrm{p}}(4)$ together with (the real part of) the approximate surface impedance $Z_{\mathrm{p}}(21)$. Somewhat similarly to Fig. 2, the exact surface impedance resembles the constant approximate surface impedance to some extent but the non-zero imaginary part of the exact impedance makes a significant difference, so the phase angle (not shown here) is far from being zero as the validity of the approximate impedance would demand. Anyway, we may obviously conclude that the layered earth structure in Japan provides an explanation for the observed correlation of GIC with the magnetic variation field. Thus, arguments related to the deep oceans and to the resulting coast effect in Japan are probably not needed.

\section{Concluding Remarks}

The surface impedance expresses the relation between the horizontal geoelectric and geomagnetic variation fields at the earth's surface. It depends on the conductivity structure of the earth. Usually, as also in this paper, the surface impedance is considered in the frequency domain. Investigations of the dependence of the surface impedance on the frequency elucidate the relation between the electric and magnetic fields in the time domain as well. In particular, if the surface impedance is independent of the frequency the electric field has exactly the same time variation as the magnetic field, and if the surface impedance has a linear dependence on the frequency the electric field follows the time derivative of the magnetic field. Observational evidence exists that in some cases the time behaviour of the geoelectric field resembles that of the geomagnetic field, and in other cases the time variations of the geoelectric field and of the time derivative of the geomagnetic field are similar. In this paper, we attack the issue in a quantitative manner by considering a two-layer earth model, which enables an analytic formula for the surface impedance.

We demonstrate that if the earth is composed of a lessconducting upper layer above a highly-conducting bottom the geoelectric field tends to follow the geomagnetic time derivative whereas a poorly-conducting bottom underlying a thin good conductor at the top of the earth results in a geoelectric field varying similarly to the geomagnetic field.

The geoelectric field drives geomagnetically induced currents (GIC) in ground-based technological networks. This process has a dc character, which means that GIC follow the time behaviour of the electric field. Thus the conclusions about the relation between the geoelectric and geomagnetic fields are directly applicable to GIC and the magnetic field.

Acknowledgments. The author wishes to thank an anonymous referee (ref. A) and Dr. László Szarka (ref. B) for very good and constructive remarks. In particular, their suggestions to relate the contents of this paper to magnetotelluric literature are gratefully acknowledged. Special thanks go to Dr. Szarka for advising the author about the paper by Berdichevsky and Dmitriev (1976) and for providing a copy of it.

\section{References}

Berdichevsky, M. N. and V. I. Dmitriev, Basic principles of interpretation of magnetotelluric sounding curves, in Geoelectric and Geothermal Studies (East-Central Europe, Soviet Asia), KAPG Geophysical Monograph, edited by A. Ádám, Akadémiai Kiadó, Budapest, Hungary, 165 
$221,1976$.

Boteler, D. H. and R. J. Pirjola, The complex-image method for calculating the magnetic and electric fields produced at the surface of the Earth by the auroral electrojet, Geophys. J. Int., 132(1), 31-40, 1998.

Boteler, D. H., R. J. Pirjola, and H. Nevanlinna, The effects of geomagnetic disturbances on electrical systems at the earth's surface, Adv. Space Res., 22(1), 17-27, 1998.

Cagniard, L., Basic theory of the magnetotelluric method of geophysical prospecting, Geophysics, 18, 605-635, 1953.

Kappenman, J. G., Geomagnetic disturbances and impacts upon power system operation, in The Electric Power Engineering Handbook, 2nd edition, edited by Leonard L. Grigsby, CRC Press/IEEE Press, Chapter 16, pp. 16-1-16-22, 2007.

Kaufman, A. A. and G. V. Keller, The Magnetotelluric Sounding Method, Methods in Geochemistry and Geophysics, 15, 595 pp., Elsevier Scientific Publishing Company, 1981.

Pirjola, R., Electromagnetic induction in the earth by a plane wave or by fields of line currents harmonic in time and space, Geophysica, 18(1-2), $1-161,1982$.

Pirjola, R. and A. Viljanen, On geomagnetically-induced currents in the Finnish $400 \mathrm{kV}$ power system by an auroral electrojet current, IEEE Trans. Power Delivery, 4(2), 1239-1245, 1989.

Pirjola, R. and A. Viljanen, Complex image method for calculating electric and magnetic fields produced by an auroral electrojet of finite length, Ann. Geophys., 16(11), 1434-1444, 1998.

Pirjola, R., D. Boteler, and L. Trichtchenko, Ground effects of space weather investigated by the surface impedance, Earth Planets Space, 61(2), 249-261, 2009.

Schmucker, U., Anomalies of geomagnetic variations in the southwestern United States, Bulletin of the Scripps Institution of Oceanography of the University of California, 13, 165 pp., La Jolla, California, USA, University of California Press, 1970.

Szarka, L., A compact representation of magnetotelluric responses for twolayer models, Geophys. Prospect., 45(5), 763-774, 1997.

Szarka, L. and G. Fischer, Electromagnetic parameters at the surface of a conductive halfspace in terms of the subsurface current distribution, Geophys. Trans., 35(3), 157-172, 1989.

Trichtchenko, L. and D. H. Boteler, Response of power systems to the temporal characteristics of geomagnetic storms, CD ROM Proceedings of the Canadian Conference on Electrical and Computer Engineering (CCECE), IEEE Ottawa, Ottawa, Canada, May 7-10, 2006, 4 pp., 2006.

Trichtchenko, L. and D. H. Boteler, Effects of recent geomagnetic storms on power systems, Proceedings of the 7-th International Symposium on Electromagnetic Compatibility and Electromagnetic Ecology, SaintPetersburg, Russia, June 26-29, 2007, 265-268, 2007.

Wait, J. R., Wave Propagation Theory, 348 pp., Pergamon Press, New York, 1981.

Watari, S., M. Kunitake, K. Kitamura, T. Hori, T. Kikuchi, K. Shiokawa, N. Nishitani, R. Kataoka, Y. Kamide, T. Aso, Y. Watanabe, and Y. Tsuneta, Measurements of geomagnetically induced current in a power grid in Hokkaido, Japan, Space Weather, 7(3), S03002, doi: 10.1029/2008SW000417, 2009.

Weaver, J. T., Mathematical Methods for Geo-electromagnetic Induction, 316 pp., Research Studies Press Ltd., Taunton, Somerset, England, John Wiley \& Sons, Inc., 1994.

Weidelt, P., The inverse problem of geomagnetic induction, Zeitschrift für Geophysik, 38, 257-289, 1972.

R. Pirjola (e-mail: risto.pirjola@fmi.fi) 